\title{
INTEGRATED MULTISENSORY ROBOTIC HAND SYSTEM FOR DEFORMABLE OBJECT MANIPULATION
}

\author{
Fouad F. Khalil, Pierre Payeur, Ana-Maria Cretu \\ School of Information Technology and Engineering, \\ University of Ottawa \\ Ottawa, ON, Canada, K1N 6N5 \\ [fkhalil, ppayeur, acretu]@site.uottawa.ca
}

\begin{abstract}
Designing a dexterous robotic hand able to interact intelligently with deformable objects constitutes a challenging area of research where many issues are yet to be solved. The complexity of such interactions requires the assistance of intelligent multisensory robotic systems that combine measurements collected from different sensors in order to accurately plan for the forces to be applied on the deformable object. This paper presents the development of a real-time multisensory robotic hand platform that incorporates live measurements of its internal position, velocity and force parameters along with data from external tactile sensors and a stereoscopic vision device. The resulting prototype of the integrated multisensory system is validated experimentally by the computation of deformable object models in which the measurements are merged. A formal dynamic model is discussed and a neural network representation model is presented. The results demonstrate the performance and suitability of the multisensory platform for the development of enhanced robotic hand capabilities when manipulating deformable objects.
\end{abstract}

\section{KEY WORDS}

Sensor fusion, deformable objects, elastic properties, dexterous manipulation.

\section{Introduction}

Future autonomous robotic systems aiming at real world applications will require more flexible and general solutions carried out by imitating the human vision and arm-hand sensorial capabilities. Human experience with objects manipulation provides a strong evidence of the ability of vision to interact with both servo-muscular and touch sensory systems in every day grasping and manipulation tasks. Autonomous robots would therefore benefit from similar coordination between these complementary sensory systems to work efficiently in unknown environments and adapt to unpredictable modifications in their working environment. Vision provides rich knowledge about the spatial arrangement, i.e. the geometry and topology of objects to be manipulated. It is well established that the grasping process can be improved by using visual feedback such that the geometrical relationship between the object and the robot end-effector is monitored. Conversely, integrating visual feedback with force/contact sensing can cope with the inaccuracy of vision systems alone due to occlusions or limited resolution, and with the inability of a vision sensor to provide force estimation.

For the major part of robotics literature, the objects to be grasped and manipulated are considered to be rigid. Until now, less effort has been made in investigating the manipulation of deformable objects. In reality, numerous objects are of such type, that is, mostly unsymmetrical, compliant, and with alterable shapes. Robot manipulation of deformable objects still offers important challenges to the robotics community and represents a major research direction for the development of the future generation of autonomous robots.

This paper presents the integration of different complementary sensors into a real-time multisensory feedback system. It provides means for modeling deformable objects from real measurements collected with a robot hand when it comes in touch with and interacts with a deformable object. Such models can then enhance the manipulation capabilities of a dexterous robotic hand. Robotic hand dexterity is a broad concept that involves aspects of, and usually a compromise between, ability and stability in performing motions of the manipulated object by hand palm and fingers [1].

The developed integrated multisensory platform combines the robotic hand's joints position and velocity measurements with force and tactile information, as well as visual shape estimation. Such multisensory feedback is essential to implement classical or modern closed-loop control algorithms to perform manipulation tasks with deformable objects that require real-time control. Otherwise, a precise model of the object's shape and deformation characteristics must be available a priori, often from approximate parametric modeling, when implementing an alternative open-loop control strategy to manipulate the object. Such open-loop control scenarios are in fact hard to implement for deformable object manipulation because the objects' physical behaviour is often dynamically changing over the course of the manipulation process. Object deformation modeling techniques frequently used in the literature are mainly based on mass-spring or finite-element modelling techniques [2]. Mass-spring models are relatively simple 
and respond faster but their structure is often application dependent. Moreover, the resulting behaviour varies dramatically with different spring configurations and its constant values lead to less accurate models that cannot efficiently account for live changes in object's characteristics. On the other hand, finite-element methods can generate more accurate models but incur heavy computational burden resulting in significant time delays, which compromises the real-time requirements of the manipulation of deformable objects.

These limitations are addressed by the multisensory solution developed in this paper in combination with adapted modeling approaches. One method consists of identifying the parameters of the classical equation of motion representing the fingertip dynamics in the joint space from actual measurements collected with the multisensory platform rather than from predetermined and approximate parameters definition. The second method proposes to use the multisensory platform with neural networks to acquire (off-line) and then predict in real-time (on-line) the complex relationship that exists between the deformation of the object and the interaction parameters resulting from the manipulation with a robot hand.

The experimental prototype of the proposed multisensory robotic hand system is composed of the three-finger Barrett hand [3], to which PPS RoboTouch tactile pads are added [4]. The integration of the tactile pads with the internal native hand sensors (encoders and strain gauges) makes it possible to measure in real-time the position of each finger, its velocity, and the force it exerts on the object, as well as the opposing reaction force from the object. Additionally, a stereoscopic vision device is part of the multisensory robotic system to simultaneously monitor and track in three dimensions the deformation of the manipulated object's contour and surface shape. This platform is integrated within the 3D virtual environment of the "GraspIt!" robotic simulator [5]. The integration of all sensing modalities provides an innovative solution and experimental platform that allows for live and comprehensive monitoring of a deformable object under manipulation by a robotic hand. It brings an significant improvement over previous work that concentrated on the modeling and control of a robotic hand device and did not include live visual monitoring of deformation nor force and tactile interaction measurements [5].

The following sections will introduce the main strategies available in the literature for the manipulation of deformable objects. Next the characteristics and modes of operation of the various sensors embedded in the multisensory system will be detailed. Then the integrated experimental test bed will be described along with the specifications and samples of measurements extracted. Finally, two alternative modeling techniques, that directly make use of the signals generated by the multisensory platform to map the force/deformation characteristics of deformable objects, will be presented as an experimental validation and demonstration of the suitability of the multisensory robotic hand system.

\section{Handling Deformable Objects}

Performing robotic manipulation on an object in an unknown configuration typically involves an initial exploratory stage assisted by vision sensors to guide the robot arm toward the object. Successful attempts have been reported in the literature to achieve effective visual feedback. The majority of these attempts focus on tracking and recovering grasp points on a non-rigid object surface in 1D or 2D using visual sensor, as well as in achieving some feedback control from those points. In an early effort, Sullivan and Papanikolopoulos [6] proposed a model-based approach for tracking of deformable objects based only on visual feedback where active contours of the object are defined.

However, when a robotic hand is used and needs to deal with deformable objects, then visual information is preferably complemented with force and/or tactile measurements, from the instant when the effector comes in contact with the surface of the object. Research efforts were recently presented to control a dexterous multifingered hand during deformable object manipulation using force/tactile feedback. In [7, 8], Minyong et al. collected and used force/tactile data to imitate the movements of the human hand. Other research directions address the resolution of the interaction control problem using multisensory feedback. Hirai et al. [9] proposed a real-time visual tracking method for the manipulation of deformable planar parts. They combine tactile and visual feedback to control the motion of a deformable object in 2D with respect to the positions of selected markers considered as reference points. Another approach to cope with 2D deformable objects using multisensory feedback was proposed by Huang et al. [10]. Their algorithm relies on high speed visual detection and visual/force sensor fusion. Other visual/force sensor fusion methods to track and control deformations in 1D, such as linear objects, and in 2D, such as planar objects, are reviewed by Henrich and Worn [11], who describe some methods in relation with specific industrial applications.

Among the few research efforts reported in the literature on monitoring 3D objects' deformation, Howard and Bekey [12] developed an off-line generalized solution to handle unknown deformable objects in $3 \mathrm{D}$. In their experiment, they used force sensors mounted on two cooperative manipulators trying to lift up an object against gravity. Force data is collected and used to approximate the 3D deformation while a stereoscopic vision is incorporated to detect if slippage occurs. The research presented in this paper addresses a different situation where the deformable object is manipulated by a robotic hand rather than by collaborative robot arms, and where the vision sensor monitors the actual 3D shape of the object, not the stability of the grasp through the detection of slippage. All measurements are merged in a same model, which goes beyond previous undertakings. 


\section{Multisensory System Components}

The multisensory platform was developed around the Barrett hand [3] which is a three-finger robotic hand, with each finger counting two joints. This section analyzes its mechanical and internal sensors characteristics in order to establish a formal correlation between the physical interaction phenomena and the raw measurements. It also compiles the experimental procedures performed to define the mathematical formulations required to properly interpret the signals generated from the hand's internal sensors. The extension achieved over the "GraspIt!" robotic virtual environment software [5] to allow for the integration of the multisensory system is also reported.

\subsection{Hand's Mechanical Modeling and Sensors Characteristics}

The outer distal joint joins the two links of a finger. The inner joint enables the finger to move in and out relative to the palm. The outer link is actuated and coupled to the inner link that can move at a fixed rate with the outer link. One of the three fingers is stationary while the other two can spread synchronously and symmetrically about the palm up to 180 degrees. As a result, despite the hand eight axes, it has four degrees of freedom, one for the spread angle and three for the angles of the inner links. The coupling between the inner and outer links for each of the three fingers is produced using a clutch setup called "Torque Switch" that makes the three-finger mechanism under-actuated and enables the motion under control of only three motors. A fourth motor is used to control the spread motion of two fingers around the palm. The significant functionality of this coupling mechanism is provided by allowing the outer link to continue to move further even though the inner link gets obstructed by an object, allowing the outer link to close in around the object. This is referred to as "breakaway".

The hand also provides built-in internal sensing capabilities to read the position and velocity measurements of its eight joints. These measurements are reported in terms of incremental encoder counts with a resolution of 360 encoder counts per revolution. The position is measured in terms of encoder counts, from fully open to fully closed, as 0 to 17000 for the fingers, and from 0 to 3140 for the spread, respectively. The outer link (fingertip) corresponding Cartesian coordinates can be calculated using the hand's forward kinematic model [3] where the distal joint angles are related to the encoder counts by a gear ratio of $1 / 375$ before breakaway, and by a gear ratio of $4 / 375$ after breakaway.

The raw velocity is measured in encoder counts per sample time, with $T_{s}=256 \mu \mathrm{sec}$, on the hand electronics. Therefore, the velocity of the joints, $v_{k}$, can be converted to revolution per second by:

$v_{k}=\frac{\text { Encoder counts per sample time }}{N * T}, \quad k \in[1 \ldots 3]$

where $N=360$ and $\mathrm{T}=2.23 \times 10^{-4} / \mathrm{T}_{\mathrm{s}}$, in seconds. The joint velocity in radians per second corresponds to 6.28 times equation (1). To compute the outer link's velocity in radian per second before and after breakaway the value should also be multiplied by $1 / 375$, and $4 / 375$, respectively. The maximum speed the hand can achieve with no load is 3162 revolutions per second.

Along with the joints angle and their velocity, force sensing capability is also available on the hand via three strain gauges, respectively installed at each finger. The force sensors measure the torque externally applied about the distal joint of the finger over a range of $-/+1 \mathrm{~N}-\mathrm{m}$, where each strain gauge measures the differential tension in the cable/tendon running through the finger to the inner joint. Therefore the strain gauge value represents the strain amount felt on the strain gauge bridge circuitry. It generates numerical value from 0 to 255 that correspond respectively to $-1 \mathrm{~N}-\mathrm{m}$ and to $+1 \mathrm{~N}-\mathrm{m}$. However, to obtain a true physical meaning, strain gauge settings have been adjusted such that the no-load value corresponds to zero torque. The strain gauges were calibrated by taking different readings over all the possible range that relates strain values ( 0 to 255$)$ to joint torque. The strain values, actually ranging from 0 to 255 are centered to read around 135 for the no-load case. The torque felt at the joint (in N$\mathrm{m})$ to strain gauge value (SG) mapping used in our experiments is given by:

$$
T_{k}=-1.1675+(0.0085 * S G), \quad k \in[1 \ldots 3]
$$

whereas the actual corresponding interaction force applied at the fingertip (in $\mathrm{kg}$ ) is mapped by the following equation:

$$
F_{A, k}=-2.2118+(0.0167 * S G), \quad k \in[1 \ldots 3]
$$

where the maximum force that the hand can accommodate is limited to $2 \mathrm{~kg}$. Equations (2) and (3) are derived from a linear interpolation among calibration data obtained experimentally.

The strain gauges practically report the physical torque exerted at the distal joint where the outer and inner links meet, which is not the measure of torque exerted by the motor. After breakaway and excluding any frictional effects, the ratio of torque exerted at the distal joint versus motor torque is $375 / 4$.

The fingertip dynamics (equation of motion) at the joint space are determined by:

$$
M(\theta) \ddot{\theta}+h(\theta, \dot{\theta})+g(\theta)=\tau-J^{T}(\theta) * R
$$

where $\theta$ is the distal joint angle and its derivatives $\dot{\theta}, \ddot{\theta}$ represent the joint velocity and acceleration, respectively, $M$ is the inertia term, $h$ is the Coriolis and centrifugal term, $g$ is the gravity term, $\tau$ is the finger drive torque and is related to the motor torque according to the gear ratio mentioned above, $R$ is the external reaction forces, which is the actual action contact force on the fingertip from the object, and $J^{T}$ is the finger's Jaccobian transpose. The latter corresponds to the length of the moment arm, i.e. the outer link (fingertip) length, which is approximately 
$50 \mathrm{~mm}$ according to mechanical specifications. Equation (4) defines the entire mechanical operation of the robot fingers and can be used to encode the characteristics of a given deformable object, via the external reaction forces, $R$, that it exerts on the fingertip during manipulation, assuming that all other parameters related to the mechanical construction of the robot hand are properly identified and remain constant.

\subsection{Integration with Robot's Virtual Environment}

The interface to the proposed multisensory robot hand system advantageously builds upon an existing software package, the robot simulator "GraspIt!" which was introduced by Miller and Allen [5]. The software is an interactive simulation, planning, analysis, and visualization virtual environment for robotic hand grasping and manipulation, and offers a partial integration with the Barrett hardware platform.

However, sophisticated grasping and manipulation strategies, especially the ones dedicated for deformable objects cannot be performed with the original version of "GraspIt!" due to the lack of suitable sensor models and interfacing with vision, force and tactile sensors. In the present research, such sensors' modalities were added to the software platform while operating in close interaction with the hardware system and taking advantage of the rendering functionalities aside the built-in robot hand model. The resulting integrated multisensory system makes it possible to plan and perform advanced control algorithms for the interaction process that meets the constraints and complexity of the deformable object case.

\section{Stereoscopic Vision Sensor}

The robotic hand platform described previously is also extended with a stereoscopic vision system that utilizes two Point Grey Research Flea 2 industrial cameras [13]. They provide non-compressed video streams via a FireWire bus (IEEE-1394b), with a resolution up to $640 \times 480$ pixels at 30 fps. However, due to the time required by the algorithm to execute, a 320x240 pixels resolution is used to achieve real-time tracking of $3 \mathrm{D}$ surface deformation. Considering the size of an object to fit in the Barrett hand, the cameras are positioned at 20 $\mathrm{cm}$ from each other, with an angle of about $30^{\circ}$ between their respective optical axes to ensure that the deformed object covers a large part of the image plane. The experimental system configuration is shown in Fig. 1.

This section describes the camera to hand calibration method used and presents the 3D estimation algorithm that is proposed for this vision sensor to extract the information necessary for 3D reconstruction of a deformable object surface in real time.

\subsection{Camera to Hand Calibration}

In order to ensure accurate and consistent merge of information with the other measurement sources, a calibration procedure between the workspace of the Barrett hand and the stereo pair has been developed. It uses a tiny $4 \times 3$ checkerboard calibration pattern which is positioned in the palm of the Barrett hand as shown in Fig. 2. The centres of the black squares in the checkerboard pattern are used as feature points, and are sorted according to their known geometrical distribution. The centres are found through thresholding, using the mean image value as the threshold, and blob analysis.

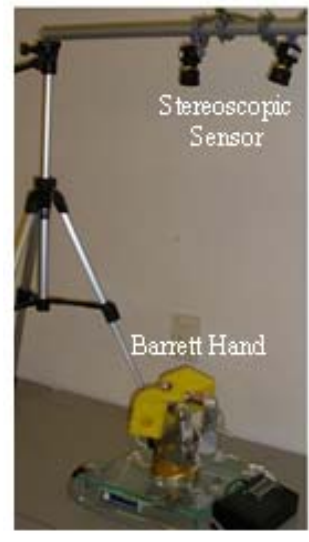

a)

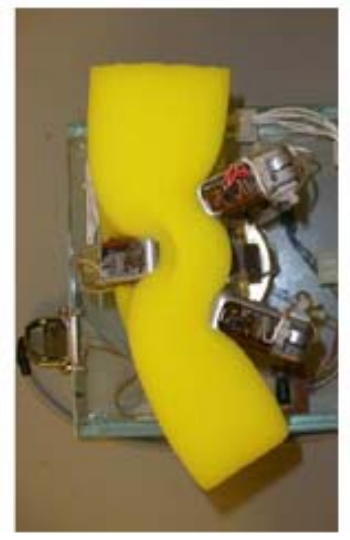

b)
Fig. 1. The system setup with stereoscopic vision sensor and Barrett hand handling a deformable object: a) lateral view, b) camera view.

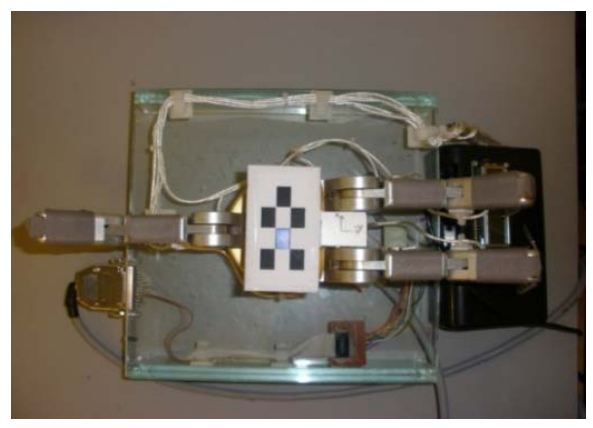

Fig. 2. Calibration checkerboard sitting in the palm of the Barrett Hand.

Once points from the checkerboard pattern are sorted for each camera in the stereo pair an implicit correspondence is assumed, and the respective $3 \mathrm{D}$ point coordinates for each corresponding set of blobs are then calculated via mid-point triangulation [14]. This provides the necessary information to extract the rotation and translation vectors of the transformation from the stereo pair to the calibration pattern. A standard transformation estimation technique that uses the relative location of feature points on the calibration target provides separately the rotation matrix and the translation vector.

\subsection{Deformable Object 3D Shape Estimation}

In order to estimate in real-time the surface shape of the manipulated deformable object, a live acquisition of $3 \mathrm{D}$ data on the surface of the object is performed using the stereo pair. Rectification of the stereo pair images is first carried out [15] and a user directed segmentation of the camera images is performed on the first frame of each 
camera's video feed by manually defining a bounding box around the region of interest. The latter is then tracked automatically over the subsequent frames with a normalized RGB colour space histogram of the object. Segmentation of the object of interest from the background is achieved with OpenCV's [16] implementation of histogram back-projection, followed by blob analysis to eliminate small objects and objects far away from the region of interest.

With the object now segmented from the image in both video feeds, Konolige's block matching stereo algorithm [17] is applied to calculate the disparity map and the corresponding 3D surface map is fed into the multisensory robotic system under the form of a 3D point cloud. The current implementation allows for a frame rate of 6 to 10 frames/sec and performs robustly on objects without explicit visual markers. The density of points reconstructed varies with the level of deformation of the object and the inherent texture of its surface [18].

\section{Incorporating Tactile Sensing}

With the stereoscopic vision system described above, it becomes possible to track the manipulated object's deformation and estimate a 3D model of its surface. However, due to occlusions that occur where the fingers are in direct contact with the object, the vision sensor fails to report complete geometrical information which is necessary to reconstruct the entire $3 \mathrm{D}$ shape of the object.

The occlusion problem is addressed by incorporating PPS RoboTouch tactile sensors [4] that can estimate the 3D local contact points between each finger and the object. This enhances the reconstructed $3 \mathrm{D}$ visual model that is displayed using the "GraspIt!" graphical interface. Seven tactile pads are mounted on the Barrett hand's three fingers and palm. Hence they cover most of the surfaces of the hand where possible contacts can be traced. Each individual pad is using capacitive-based pressure sensing technology. The pads geometry is made of a 22-element grid at $6 \times 6 \mathrm{~mm}$ resolution installed over each finger outer link (fingertip). A separate 24-element grid at 6x6 mm resolution is mounted over each finger inner link, and a 24-element grid at $10 \times 10 \mathrm{~mm}$ resolution is set on the palm. The pressure data from the entire set of 162 tactile elements distributed over the seven pads is scanned at a $30 \mathrm{~Hz}$ sampling rate. These pads are used to give the Barrett robotic hand a "sense of touch" by calculating the pressure sensed in contact areas. As a result, reaction forces from the object side opposing the force exerted by the fingers are estimated in real-time. This supplementary information provides all the necessary components to resolve the classical Newton's second law, equation (4). The latter can then be used to encode a given object's elastic properties collected via an experimental identification phase. The resulting model of the deformable object then serves to derive the required control law that governs the interaction process of the hand with the object.
The tactile sensor output is a number of counts that reflects the amount of pressure imposed on each of the 162 tactile elements. Each element generates a 15-bit signal over the range $0-32768$. In order to convert the raw data to physical units, a calibration process must be performed on the tactile elements to directly relate a pressure value in $\mathrm{kPa}$ units to the measured raw data. The calibration data used here are provided by the sensor manufacturer. A linear interpolation between seven calibration points that best fits a straight line over the calibration measurements is performed to obtain the physical equivalent pressure. However, the relationship between counts and the physical units varies from element to element. Therefore, a separate linear regression is used to calculate the parameters, $a_{i}$ and $b_{i}$, for each of the 162 interpolations, each representing one tactile pad element:

$$
P_{i}=a_{i} * \text { count }_{i}+b_{i}, \quad i \in[1 \ldots 162]
$$

The resulting parameters are encoded in a look-up table as part of the integrated multisensory robotic hand system that reads the raw count $t_{i}$ values in real time. Using the predefined array of parameters, $a_{i}$ and $b_{i}$, equation (5) converts the count $_{i}$ value reported by the $i^{\text {th }}$ tactile element into a live equivalent pressure, $P_{i}$, in $\mathrm{kPa}$. Then this pressure is multiplied by the size of the active contact area, $A_{i}$, in square $\mathrm{cm}$ in order to convert the pressure into reaction force units, $F_{R, k}$, over a given fingertip and expressed in Newton, or equivalently in $\mathrm{kg}$ after dividing by the gravitational acceleration constant, $g$.

$$
F_{R, k}=\sum_{i \in k} P_{i} * A_{i}, \quad k \in[1 \ldots 3]
$$

\section{Experimentation with the Multisensory Robotic System Prototype}

The resulting integrated multisensory robotic hand system is composed of a Barrett hand unit with built-in sensors to acquire the position, velocity, and torque measurements (Section 3.1) along with the local reaction force data collected from the tactile pads mounted on the hand fingers and palm (Section 5). A stereoscopic camera is installed perpendicularly to the surface of the object being manipulated to reconstruct its surface shape (Section 4).

\subsection{Collecting Multisensory Samples}

As shown in Figs. $1 \mathrm{~b}$ and $3 \mathrm{~b}$, the hand is positioned with its palm up, facing the cameras, to allow visual monitoring of the interaction with a minimum of occlusions. All interaction parameters (positions, velocities, torques, reaction forces and $3 \mathrm{D}$ shape) are collected simultaneously for all three fingers using the multisensory system software interface that is built as an extension to the "GraspIt!" virtual simulator environment. The latter is used to render a synchronized 3D mesh visual representation of the object's shape, as illustrated in Fig. 3c, that is computed in real-time with a 
Delaunay triangulation between the reconstructed 3D points. Simultaneous data acquisition from all sensors relies on multiple threads that are synchronised with a Qt timer [19], a software component that was added to the "GraspIt!" software platform in order to create the multisensory robotic hand system.

Deformable objects that were considered in this round of experiments are pieces of foam of various shapes and colours, and that exhibit different elastic characteristics. Figs. 1 and 3a show two of the objects used in the experiments. Fig. $3 \mathrm{~b}$ shows an object manipulated by the hand at a certain stage of the compression test performed to identify the object's properties. Fig. 3c illustrates the corresponding 3D reconstruction estimated with the stereovision component of the multisensory system and displayed with the "GraspIt!" virtual environment interface from various angles and with different scales.

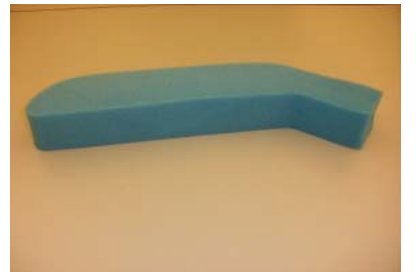

a)

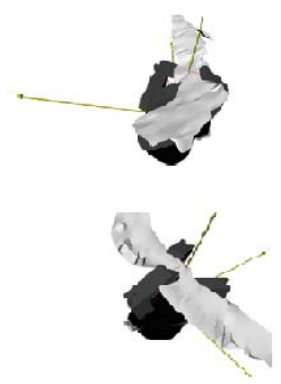

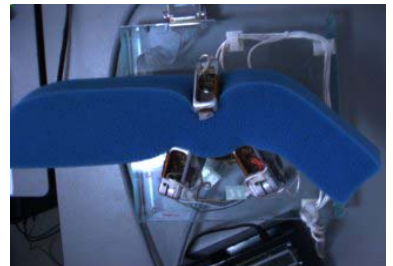

b)

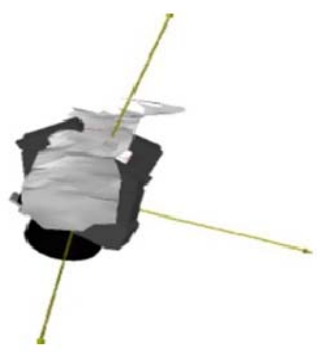

c)

Fig. 3. a) A sample foam object, b) the object manipulated by the hand while applying forces, and c) the corresponding live mesh representation of the estimated 3D surface shape from different views.

All interaction parameters are acquired for every finger at every sampling instant. The parameters corresponding to each finger motion are the position, Fig. $4 \mathrm{a}$, and velocity, Fig. 4b. The two motion measurements are represented as the number of pulses in the encoder that reads the angle of the motor that drives the finger. These measurements, using the raw motor encoder data, provide a compact representation of the hand motion in its workcell. This mapping has a beneficial impact on the update rate of the control law implementation during live manipulation with multisensory feedback, as provided by the proposed platform. Raw measurements can also be converted into the equivalent Cartesian coordinates using the hand kinematic model [3] for physical interpretation.

The measurements of the torques (Fig. 4c) and reaction forces (Fig. 4d) are collected simultaneously at each finger from the strain gauges and the tactile pads, respectively. The torque measurements are related to the driving motor torques, as detailed in Section 3.1. When collected during the identification phase together with the motion and velocity measurements on a given deformable object, the torque and reaction force data provide data sets to resolve the hand and interaction dynamics, equation (4). This model can therefore be used as a physical representation of the deformable object's elastic properties and a controller can be tuned to optimise manipulation performance, stability and integrity, over that object. An alternative model of the deformable object that makes use of the same multisensory data sets will also be proposed in Section 7.

\subsection{Identification of an Object's Elastic Properties}

In order to conduct a proper identification of an object's elastic properties using the multisensory robotic hand system, it is considered that the object has been grasped already by the three fingers. The balanced grasping forces to maintain the object within the hand grip without slippage are initially applied. The hand then compresses the object repetitively by contracting and relaxing its grip over a period of several sampling periods. A number of 18 samples/sec were collected in our experiments. The test is carried out using Barrett hand's real-time mode which allows the input fed to the hand system to generate a periodic movement of the fingers in order to properly excite the object and extract its dynamic characteristics. The hand's three fingers motion are defined to follow a sinusoidal profile defined in encoder pulses to cover a proper range of compression of the object.

$$
m_{k}=10+40 \sin (3000 t), \quad k \in[1 \ldots 3]
$$

Fig. 4a-c show the resulting position, $p_{k}$, velocity, $\mathrm{v}_{k}$, and corresponding torque values, $T_{k}$, respectively, measured on the three fingers holding the deformable object of Fig. 3a during the identification procedure. Fig. $4 \mathrm{~d}$ shows the reaction forces, $F_{R, k}$, as perceived by the tactile sensors over the three outer links (fingertips). The tactile data collected from the active tactile elements are lumped to produce one reaction force acting on each fingertip, as in equation (6).

The position profile of the hand (Fig. 4a) shows the actual compression of the object while identification is carried out by applying forces resulting from the torques shown in Fig. 4c. During the procedure, fingers 1 and 2 are penetrating more deeply inside the object material while finger 3 acts as the thumb to maintain a stable grasp, leading to displacement and torques of lower magnitude. It is also clear from the reaction forces measured, Fig. 4d, that the compression pulses become narrower and sharper as the fingers penetrate deeper inside the object. This indicates that the object increases its resistance against the applied force, which in fact, is characteristic of the compliance of the object. The velocity profile, as shown in Fig. $4 \mathrm{~b}$, indicates that the fingers movement also becomes slower due to increasing opposing reactions from the object as the fingers get deeper into it. 


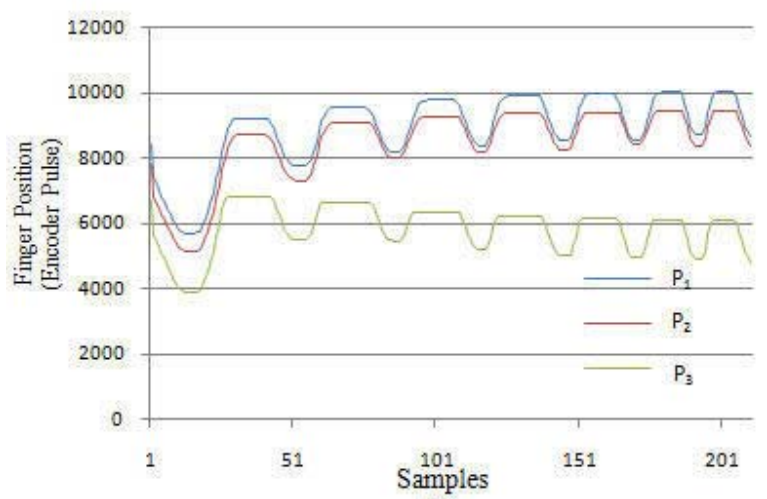

(a)

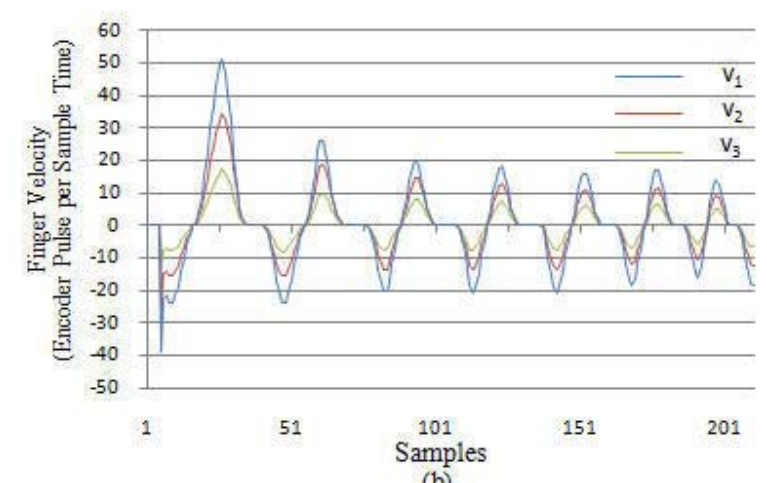

(b)

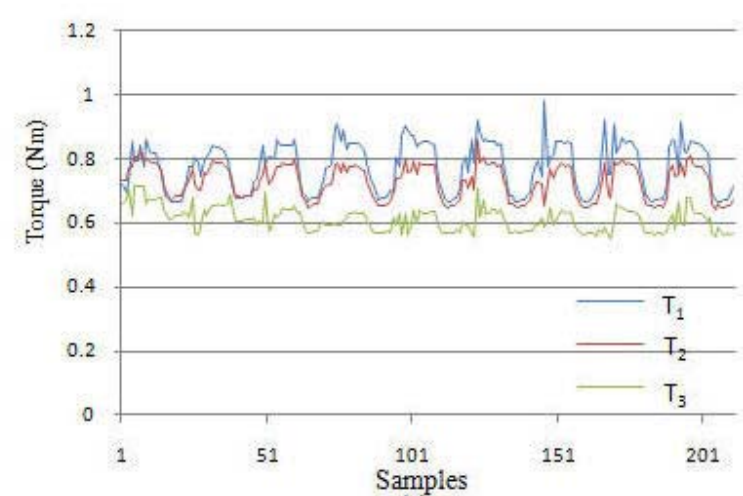

(c)

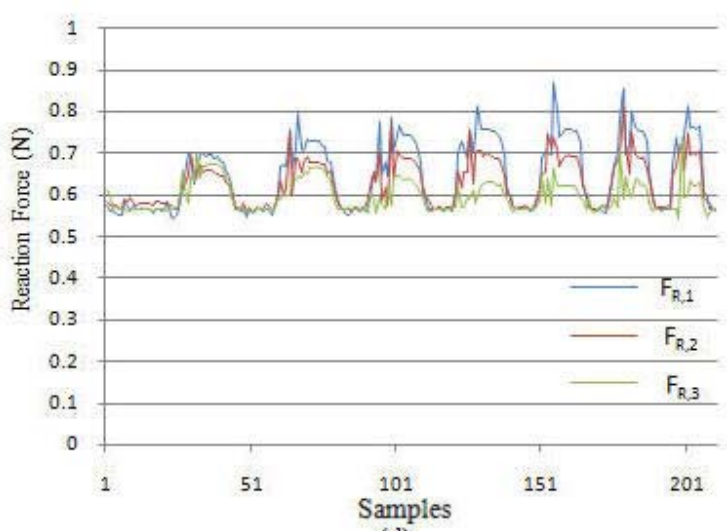

(d)

Fig. 4. Measurements collected over the elasticity identification procedure: a) fingers positions in encoder pulses, b) velocities in encoder pulses per sample time, c) applied torques in N-m, and d) opposing reaction forces in $\mathrm{N}$.
In parallel with the collection of the mechanical parameters, the vision sensor extracts and estimates in real-time the 3D location of points over the object surface. It then generates a 3D mesh that dynamically follows the evolution of the shape of the surface of the object, which provides rich information about the deformation experienced by the object under the effect of the applied forces during the identification phase. The modeling framework proposed in the next section makes extensive use of this data to generate a comprehensive representation of the object's elastic properties.

\section{Deformable Objects Properties Estimation with Neural Networks}

Making use of the integrated multisensory system, a distinct research direction that is pursued is the formal mapping of objects' elastic properties, by means of neural networks, using actual measurements. Such models are essential to predict the shape of real deformable objects under robotic manipulation. Due to their intrinsic nonlinearity, their computational simplicity and their ability to learn, neural networks are natural candidates for such modeling tasks. In an initial study [20], we successfully correlated the finger positions in encoder pulses $\left(p_{1}, p_{2}\right.$, $\left.p_{3}\right)$, shown in Fig. $4 \mathrm{a}$, and the torques on each finger $\left(T_{1}\right.$, $T_{2}, T_{3}$ ), as defined in equation (2) and shown in Fig. 4c, corresponding to the applied interaction forces, with the shape information in form of the contour of the deformable object. In this experiment, the contours were extracted from a sequence of images collected from the camera and using a feedforward neural network architecture.

The relationships between fingers positions, the torques leading to applied interaction forces and the object contour shape are mapped in a separate network. The latter has six input neurons associated with the interaction parameters $\left(p_{1}, p_{2}, p_{3}, T_{1}, T_{2}, T_{3}\right), 30$ hidden neurons, and the output vector is the set of coordinates for the $n$ points on the contour that the object exhibits under the current interaction configuration as shown in Fig. 5. One network is used for each deformable object as it encodes its intrinsic elastic properties.

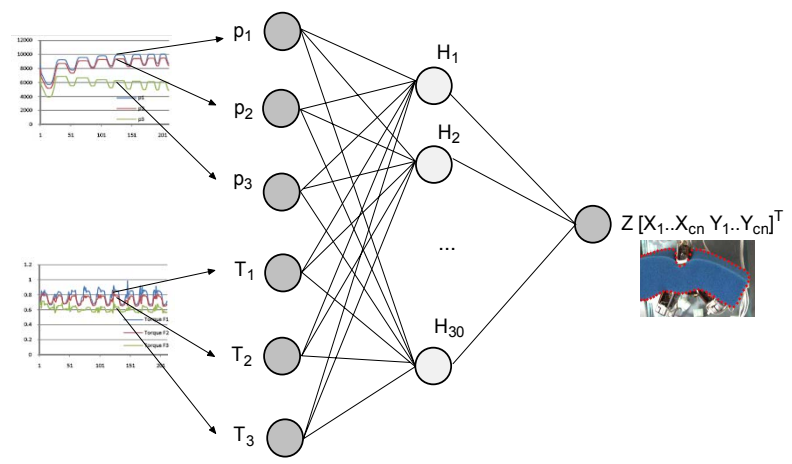

Fig. 5. Neural network architecture used to map the robot hand's fingers positions and applied torques to contour deformation of a deformable object for encoding its elastic properties. 
Each network is trained using the data sets generated by the proposed multisensory robotic system during the identification procedure of the deformable object properties, as detailed in Section 6.2. The benefits of this modeling approach are that: $i$ ) it directly uses experimental measurements made available from the proposed multisensory robotic hand system, $i i)$ it captures the shape of the deformable object under study and its dynamics under deformation, and iii) it can also predict in real-time the object's expected behaviour under previously unrecorded interactions, without imposing particular material models, as often required with other representations. The latter feature is particularly suitable to plan and control the operation of a robotic hand involved in the dexterous manipulation of deformable objects with nonlinear properties.

\section{Conclusion}

To better achieve dexterity in the robotic manipulation of deformable objects, robots should ultimately produce similar adaptive sensorial coordination as human beings do (i.e. vision, servo and touch capabilities). This would make robots more effective in unknown and uncalibrated environments and therefore provide them with the capability to adapt their behavior to unpredictable modifications. Based on the measurements provided by an integrated multisensory feedback system about the object in contact with a robot hand, control strategies can be progressively tuned and adapted in an autonomous manner during the task execution phase.

This paper presents an integrated multisensory feedback system developed around the Barrett hand that can monitor in real time all factors influencing an object deformation during manipulation. Features extracted by a vision sensor are used to build a 3D live model of the surface deformation. This model is enhanced by merging synchronously collected measurements on fingers position, velocity, torque and tactile reaction forces. A procedure for experimental identification of an object's elastic properties is introduced that builds upon the available measurements. Suitable modeling frameworks are also proposed to encode the complex dynamic behaviors of deformable objects under manipulation by a robot hand. The resulting system aims at providing robotic researchers with a complete and fully integrated platform to perform experimentation and further develop dexterous robotic interaction technologies.

\section{Acknowledgement}

The authors acknowledge the financial support from the Natural Sciences and Engineering Research Council of Canada to this research.

\section{References}

[1] A. Bicchi, Hands for dexterous manipulation and robust grasping: a difficult road towards simplicity, IEEE Trans. on Robotics and Automation, 16, 2000, 652-662.

[2] S.F. Gibson and B. Mitrich, A survey of deformable models in computer graphics, Technical Report TR-97-19, Mitsubishi Electric Research Laboratories, Cambridge, MA. 1997.

[3] ---, Barrett BH8 Robotic Hand. http://www.barrett.com/

[4] ---, PPS RoboTouch Sensors,

http://www.pressureprofile.com/

[5] A. T. Miller and P. K. Allen, Graspit! a versatile simulator for robotic grasping, Robotics \& Automation Magazine, vol 11, 2004, 110-122.

[6] M. J. Sullivan and N. P. Papanikolopoulos, Using activedeformable models to track deformable objects in robotic visual servoing experiments, Proc. IEEE Int. Conf. on Robotics and Automation, 1996, 2929-2934.

[7] P. Minyong, K. Mouri, H. Kitagawa, T. Miyoshi, and K. Terashima, Hybrid impedance and force control for massage system by using humanoid multi-fingered robot hand, Proc. IEEE Int. Conf. on Systems, Man and Cybernetics, 2007, 30213026.

[8] K. Mouri, K. Terashima, P. Minyong, H. Kitagawa, and T. Miyoshi, Identification and hybrid impedance control of human skin muscle by multi-fingered robot hand, Proc. IEEE/RSJ Int. Conf. on Intelligent Robots and Systems, 2007, 2895-2900.

[9] S. Hirai, T. Tsuboi, and T. Wada, Robust grasping manipulation of deformable objects, Proc. IEEE Int. Symp. on Assembly and Task Planning, 2001, 411-416.

[10] J. Huang, I. Todo, and T. Yabuta, Position/force hybrid control of a manipulator with a flexible tool using visual and force information, Cutting Edge Robotics, V. Kordic, A. Lazinica, and M. Merdan, Eds., (Pro Literatur Verlag, Germany), 2005, 611-628.

[11] D. Henrich and H. Worn, Eds., Robot manipulation of deformable objects, (Springer-Verlag, London, 2000).

[12] A. H. Howard and G. A. Bekey, Intelligent learning for deformable object manipulation, J. of Autonomous Robots, 9, 2000, 51-58.

[13] ---, Point Grey Research Flea2 Cameras, http://www.ptgrey.com/

[14] E. Trucco and A. Verri, Introductory techniques for $3 D$ computer vision, (Prentice Hall, 1998).

[15] A. Fusiello, E. Trucco, and A. Verri, A compact algorithm for rectification of stereo pairs, Machine Vision and Applications, Springer-Verlag, 12, 2000, 16-22.

[16] G. Bradski and A. Kaehler, Learning OpenCV, (O'Reilly, 2008).

[17] K. Konolige, Small vision systems: hardware and implementation, Proc. Int. Symp. on Robotics Research, Hyayma, Japan, 1997, 111-116.

[18] F. F. Khalil, P. Curtis, P. Payeur, Visual monitoring of surface deformations on objects manipulated with a robotic hand, Proc. IEEE Int. Workshop on Robotic and Sensors Environments, Phoenix, AZ, 2010.

[19] J. Blanchette and M. Summerfield, $C++\quad G U I$ Programming with Qt4, $2^{\text {nd }}$ Edition, (Prentice Hall, 2008).

[20] A.-M. Cretu, P. Payeur, E. M. Petriu, and F. F. Khalil, Estimation of Deformable Object Properties from Shape and Force Measurements for Virtualized Reality Applications, Proc. IEEE Int. Symp. on Haptic, Audio-Visual Environments and Games, Phoenix, AZ, 2010. 